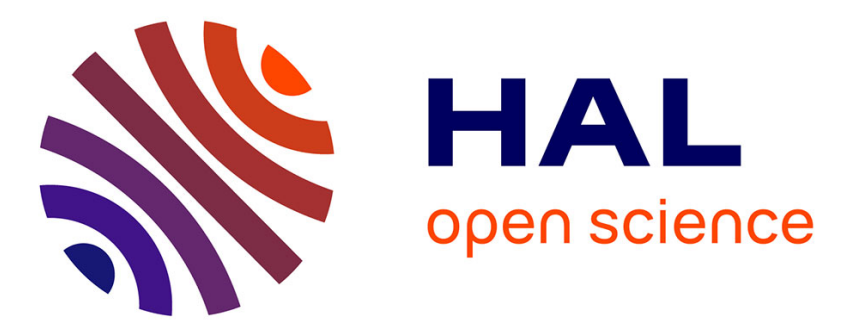

\title{
Two-scale approach to the homogenization of membrane photonic crystals
}

D. Felbacq, Guy Bouchitté, B. Guizal, A. Moreau

\section{To cite this version:}

D. Felbacq, Guy Bouchitté, B. Guizal, A. Moreau. Two-scale approach to the homogenization of membrane photonic crystals. Journal of Nanophotonics, 2008, 2 (1), pp.023501. 10.1117/1.2884039 . hal-00255910

\section{HAL Id: hal-00255910 https://hal.science/hal-00255910}

Submitted on 22 Apr 2021

HAL is a multi-disciplinary open access archive for the deposit and dissemination of scientific research documents, whether they are published or not. The documents may come from teaching and research institutions in France or abroad, or from public or private research centers.
L'archive ouverte pluridisciplinaire HAL, est destinée au dépôt et à la diffusion de documents scientifiques de niveau recherche, publiés ou non, émanant des établissements d'enseignement et de recherche français ou étrangers, des laboratoires publics ou privés.

\section{(c)(1)}

Distributed under a Creative Commons Attribution| 4.0 International License 


\title{
Two-scale approach to the homogenization of membrane photonic crystals
}

\author{
Didier Felbacq, ${ }^{a}$ Guy Bouchitté, b Brahim Guizal, ${ }^{c}$ Antoine Moreau ${ }^{d}$ \\ ${ }^{\text {a}}$ University of Montpellier II, GES UMR 5650, Bat. 21 CC074 Place E. Bataillon, 34095 \\ Montpellier Cedex 05, France \\ dfelbacq@univ-montp2.fr \\ ${ }^{b}$ University of Toulon, ANAM, BP 132, 83957 La Garde Cedex, France \\ ${ }^{c}$ University of Franche-Comté, FEMTO-ST, route de Gray, 25000 Besançon, France \\ d University of Clermont II, LASMEA UMR 6602, Complexe des Cézeaux 63177 Aubière \\ Cedex, France
}

\begin{abstract}
Wave propagation and diffraction in a membrane photonic crystal with finite height were studied in the case where the free-space wavelength is large with respect to the period of the structure. The photonic crystals studied are made of materials with anisotropic permittivity and permeability. Use of the concept of two-scale convergence allowed the photonic crystals to be homogenized.
\end{abstract}

Keywords: homogenization, photonic crystals, metamaterials, effective properties.

\section{INTRODUCTION}

Photonic crystals, i.e. dielectric or metallic artificial periodic structures, are generally thought of as strongly scattering devices, displaying photonic band gaps. However, their actual electromagnetic behavior when the free-space wavelength is large with respect to the period is also interesting, because it can produce strongly anisotropic behaviors, plasmon frequencies, or even negative index materials [1]. The study of the properties of these structures in this asymptotic regime comes under the theory of homogenization [2-4]. A lot of results are by now well established both for 2D and 3D structures (even for non periodic structures [5-7]).

In this paper, we consider a photonic crystal made of a collection of parallel finite-size fibers embedded in a matrix. This covers the case of structures made out of a layer of bulk materials in which holes are made periodically (membrane photonic crystal) but also the case of structures made out of nanopillars (pillar photonic crystal $[8,9]$ ). The fibers are not supposed to be invariant in the direction of their axis (for instance they can be cone-shaped, see fig. 1). Our point is to derive the effective permittivity and permeability tensors of this structure when the ratio between the period of the structure and the free-space wavelength of the incident field is very small. We had already derived rigorous results for infinitely long cylindrical fibers [3], for which explicit formulas can be derived in some cases [2,10-12]. Here we shall get homogenized permeability and permittivity tensors with a dependence along the axis of the fibers. Let us note that our results hold for dispersive and lossy materials (it applies to imperfect metals as well as to good insulators).

\section{TWO-SCALE APPROACH TO HOMOGENIZATION}

\subsection{Description of the structure and methodology}

We consider a 2D photonic crystal such as that in fig. 1. It is constructed from a basic cell $\tilde{Y}$ pictured in fig. $2\left(\widetilde{Y}=Y \times(-L, L)\right.$, where $\left.Y=(-1 / 2,1 / 2)^{2}\right)$. A contraction ratio $\eta$ is applied to obtain a contracted cell in the horizontal directions $\left(\widetilde{Y}_{\eta}=\eta^{2} Y \times(-L, L)\right)$. In the units of the free-space wavelength, the period of the lattice is thus $\eta$. The cells are contained 
in a cylindrical domain $\Omega(\Omega=\mathcal{O} \times(-L, L))$ (cf. fig.1). The domain $\Omega$ is thus periodically filled with contracted cells. The space coordinates are denoted by $\mathbf{x}=\left(x_{1}, x_{2}, x_{3}\right)$ and we also

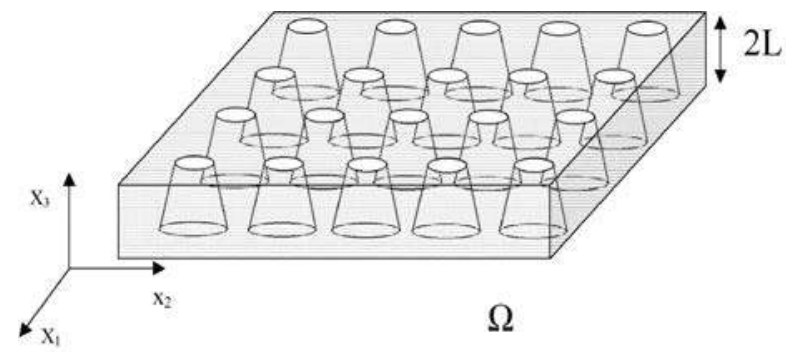

Fig. 1. Schematic of the photonic crystal.

denote $\mathbf{x}_{\perp}=\left(x_{1}, x_{2}\right)$. The coordinates in $Y$ are denoted by $\mathbf{y}=\left(y_{1}, y_{2}\right)$. We consider time harmonic fields, the time dependence is chosen to be $\exp (-i \omega t)$. For a given monochromatic incident field $\left(\mathbf{E}^{i}, \mathbf{H}^{i}\right)$, we denote by $\left(\mathbf{E}^{\eta}, \mathbf{H}^{\eta}\right)$ the total electromagnetic field.

Our aim is to pass to the limit $\eta \rightarrow 0$ and determine the limit of the couple $\left(\mathbf{E}^{\eta}, \mathbf{H}^{\eta}\right)$. In our methodology, we get at the limit a true electromagnetic scattering problem, for a given free-space wavelength $\lambda$ and a bounded obstacle $\Omega$ characterized by some permittivity and permeability tensors. This situation is quite different from other homogenization techniques, making use of periodization conditions, in which the frequency tends to zero, thus not leading to a diffraction problem but rather to an electrostatic one. Such an approach can sometimes give useful explicit formulas but generally leads to complicated formulations [10-12, 20,21]. Moreover, it does not handle the boundary effects which in some cases may lead to some miscomprehensions [13]. The relative permittivity tensor $\underline{\underline{\varepsilon}}^{\eta}(\mathbf{x})$ and relative permeability tensor

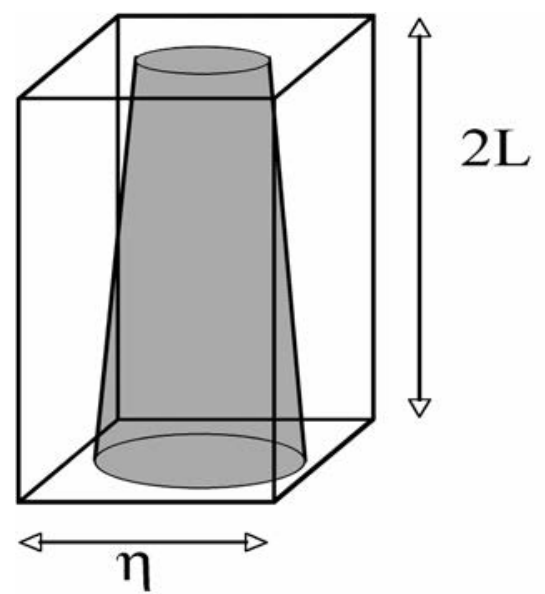

Fig. 2. Schematic of the basic cell.

$\underline{\mu}^{\eta}(\mathbf{x})$ are described by:

$$
\begin{cases}\underline{\underline{\varepsilon}}^{\eta}(\mathbf{x})=1, \underline{\mu}^{\eta}(\mathbf{x})=1 & \text { for } \mathbf{x} \in \mathbb{R}^{3} \backslash \Omega \\ \underline{\underline{\varepsilon}}^{\eta}(\mathbf{x})=\underline{\underline{\varepsilon}}^{0}\left(\frac{\mathbf{x} \perp}{\eta}, x_{3}\right), \underline{\underline{\mu}}^{\eta}(\mathbf{x})=\underline{\underline{\mu}}^{0}\left(\frac{\mathbf{x} \perp}{\eta}, x_{3}\right) & \text { for } \mathbf{x}_{\perp} \in \Omega\end{cases}
$$


where at fixed $x_{3}$, the applications $\mathbf{y} \rightarrow \underline{\underline{\varepsilon}}^{0}\left(\mathbf{y}, x_{3}\right)=\left(\varepsilon_{i j}^{0}\left(\mathbf{y}, x_{3}\right)\right)$ and $\mathbf{y} \rightarrow \underline{\underline{\mu}}^{0}\left(\mathbf{y}, x_{3}\right)=$ $\left(\mu_{i j}^{0}\left(\mathbf{y}, x_{3}\right)\right)$ are $Y$-periodic $3 \times 3$ matrix functions.

The total electromagnetic field $\left(\mathbf{E}^{\eta}, \mathbf{H}^{\eta}\right)$ satisfies

$$
\left\{\begin{array}{l}
\operatorname{curl} \mathbf{E}^{\eta}=i \omega \mu_{0} \underline{\bar{\mu}}^{\eta} \mathbf{H}^{\eta} \\
\operatorname{curl} \mathbf{H}^{\eta}=-i \omega \varepsilon_{0} \underline{\underline{\varepsilon}}^{\eta} \mathbf{E}^{\eta}
\end{array}\right.
$$

and $\left(\mathbf{E}^{s, \eta}, \mathbf{H}^{s, \eta}\right)=\left(\mathbf{E}^{\eta}-\mathbf{E}^{i}, \mathbf{H}^{\eta}-\mathbf{H}^{i}\right)$ satisfies Silver-Müller radiation conditions:

$$
\lim _{\|\mathbf{x}\| \rightarrow+\infty}\|\mathbf{x}\|\left(Z_{0} \mathbf{H}^{s, \eta} \times \frac{\mathbf{x}}{\|\mathbf{x}\|}-\mathbf{E}^{s, \eta}\right)=0
$$

where $Z_{0}$ is the impedance of vacuum.

\subsection{Derivation of the microscopic equations}

\subsubsection{A short account of the two-scale expansion}

In order to describe this problem, we will rely on a two-scale expansion of the fields. That way, the physical problem is described by two variables: a macroscopic one $\mathbf{x}$ and a microscopic one $\mathbf{y}$ representing the rapid variations of the material at the scale of one basic cell, measured by $\eta$. By noticing that there are no rapid variations in the vertical direction $x_{3}$, the microscopic variable is set to be: $\mathbf{y}=\mathbf{x}_{\perp} / \eta$. Differential operators with respect to variable $\mathbf{y}$ are denoted with a subscript $y$. The fields are periodic with respect to that microscopic variable (this corresponds to the neighborhood of the center of the first Brillouin zone). The limit problem obtained by letting $\eta$ tend to 0 , will then depend on the macroscopic, physical, variable $\mathbf{x}$ but also on the microscopic, hidden, variable $\mathbf{y}$. The total limit fields will read $\mathbf{E}^{0}(\mathbf{x}, \mathbf{y})$ and $\mathbf{H}^{0}(\mathbf{x}, \mathbf{y})$ and the observable, physical, fields will be given by the mean value over the hidden variable $\mathbf{y}$ : $\mathbf{E}(\mathbf{x})=|Y|^{-1} \int_{Y} \mathbf{E}^{0}(\mathbf{x}, \mathbf{y}) d \mathbf{y}$ and $\mathbf{H}(\mathbf{x})=|Y|^{-1} \int_{Y} \mathbf{H}^{0}(\mathbf{x}, \mathbf{y}) d \mathbf{y}$, where $|Y|$ is the measure area of $Y$. In order to lighten the notations, we denote by brackets the averaging over $Y$, hence $\mathbf{H}(\mathbf{x})=\left\langle\mathbf{H}^{0}\right\rangle$ and $\mathbf{E}(\mathbf{x})=\left\langle\mathbf{E}^{0}\right\rangle$.

The main mathematical tool that we use is a mathematically rigorous version of the multiscale expansion, widely used in various areas of physics. More precisely, for a vector field $\mathbf{F}^{\eta}$ in $\left(L^{2}(\Omega)\right)^{3}$, we say, by definition, that $\mathbf{F}^{\eta}$ two-scale converges towards $\mathbf{F}^{0}$ if for every sufficiently regular function $\phi(\mathbf{x}, \mathbf{y}), Y$-periodic with respect to $\mathbf{y}$, we have:

$$
\int_{\Omega} \mathbf{E}^{\eta}(\mathbf{x}) \cdot \phi\left(\mathbf{x}, \mathbf{x}_{\perp} / \varepsilon\right) d \mathbf{x} \rightarrow \iint_{\Omega \times Y} \mathbf{E}^{0}(\mathbf{x}, \mathbf{y}) \cdot \phi(\mathbf{x}, \mathbf{y}) d \mathbf{x} d \mathbf{y}
$$

as $\eta$ tends to 0 .

The limit field $\mathbf{F}^{0}$ is square integrable with respect to both variables $\mathbf{x}$ and $\mathbf{y}$ and is $Y$ periodic in the $\mathbf{y}$ variable (this is the definition of the space $L^{2}\left(\Omega ;\left(L_{\text {per }}^{2}(Y)\right)^{3}\right)$ ). A complete analysis of this new mathematical tool can be found in [14].

We make the physically reasonable assumption that the electromagnetic energy remains bounded when $\eta$ tends to 0 , which is equivalent to assume that $\left(\mathbf{E}^{\eta}, \mathbf{H}^{\eta}\right)$ are both locally square integrable. Then it is known [14] that $\left(\mathbf{E}^{\eta}, \mathbf{H}^{\eta}\right)$ two-scale converges towards limit fields $\left(\mathbf{E}^{0}, \mathbf{H}^{0}\right)$. This physical assumption could be justified mathematically, however it seems quite obvious, from the point of view of physics, that the limit fields exist. The point is then to give the system of equations that is satisfied by these fields and to derive the effective permittivity and permeability tensors. 


\subsubsection{The equations at the microscopic scale}

First of all, we have to determine the set of equations that are microscopically satisfied, that is, satisfied with respect to the hidden variable $y$, for that will give the constitutive relations of the homogenized medium. Multiplying Maxwell-Faraday equation by a regular test function $\phi\left(\mathbf{x}, \frac{\mathbf{x}_{\perp}}{\eta}\right)$, and integrating over $\Omega$, we obtain:

$$
\int_{\Omega} \mathbf{E}^{\eta}(\mathbf{x}) \cdot\left[\operatorname{curl}_{x}(\phi)+\frac{1}{\eta} \operatorname{curl}_{y}(\phi)\right] d \mathbf{x}=i \omega \mu_{0} \int_{\Omega} \underline{\mu}^{\eta}(\mathbf{x}) \mathbf{H}^{\eta}(\mathbf{x}) \phi\left(\mathbf{x}, \mathbf{x}_{\perp} / \eta\right) d \mathbf{x} .
$$

Multiplying by $\eta$ and letting $\eta$ tend to 0 , we get using (4):

$$
\iint_{\Omega \times Y} \mathbf{E}^{0}(\mathbf{x}, \mathbf{y}) \cdot \operatorname{curl}_{y}(\phi) d \mathbf{x} d \mathbf{y}=0 .
$$

This is equivalent to:

$$
\iint_{\Omega \times Y} \operatorname{curl}_{y} \mathbf{E}^{0}(\mathbf{x}, \mathbf{y}) \cdot \phi(\mathbf{x}, \mathbf{y}) d \mathbf{x} d \mathbf{y}=0
$$

which is nothing else but the variational form for: $\operatorname{curl}_{y} \mathbf{E}^{0}=0$. In a very similar way, but using now Maxwell-Ampere equation, we get $\operatorname{curl}_{y} \mathbf{H}^{0}=0$. On the other hand, since $\underline{\underline{\varepsilon}}^{\eta} \mathbf{E}^{\eta}$ is diver-

gence free, we have, for every test function $\phi(\mathbf{x}, \mathbf{y}), \int_{\Omega} \underline{\underline{\varepsilon}}^{\eta}(\mathbf{x}) \mathbf{E}^{\eta}(\mathbf{x}) \cdot\left[\nabla_{x} \phi+\frac{1}{\eta} \nabla_{y} \phi\right] d \mathbf{x}=0$. Multiplying by $\eta$ and letting $\eta$ tend to 0 , we get:

$$
\iint_{\Omega \times Y}{\underline{\varepsilon^{0}}}^{=}\left(\mathbf{y}, x_{3}\right) \mathbf{E}^{0}(\mathbf{x}, \mathbf{y}) \cdot \nabla_{y} \phi d \mathbf{x} d \mathbf{y}=0
$$

which can be written as (notice that the $\operatorname{div}_{y}$ operator acts only on the transverse components):

$$
\operatorname{div}_{y}\left(\underline{\underline{\varepsilon}}^{0} \mathbf{E}^{0}\right)=0 .
$$

Similarly, since the magnetic field is divergence free, we derive:

$$
\operatorname{div}_{y}\left(\underline{\underline{\mu}}{ }^{0} \mathbf{H}^{0}\right)=0 .
$$

Summing up, we have the following microscopic equations, holding inside the basic cell $Y$ :

$$
\left\{\begin{array}{l}
\operatorname{div}_{y}\left(\underline{\underline{\mu}}^{0} \mathbf{H}^{0}\right)=0 \\
\operatorname{curl}_{y} \mathbf{H}^{0}=0
\end{array},\left\{\begin{array}{l}
\operatorname{div}_{y}\left(\underline{\varepsilon}^{0} \mathbf{E}^{0}\right)=0 \\
\operatorname{curl}_{y} \overline{\mathbf{E}}^{0}=0
\end{array}\right.\right.
$$

\subsection{Derivation of the homogenized parameters}

The systems in (11) are respectively of electrostatic and magnetostatic types. This means that, with respect to the hidden variable $\mathbf{y}$, the electric field and magnetic field satisfy the static Maxwell system. This property is true only at that scale and not at the macroscopic scale. However, it is these static equations that determine the effective permittivity and permeability. Indeed let us analyze this system starting with the electric field. From the curl relation, we get $\nabla_{y} E_{3}^{0}=0$, and so $E_{3}^{0}(\mathbf{x}, \mathbf{y}) \equiv E_{3}(\mathbf{x})$. Besides, the basic cell having the geometry of a flat torus, we get the existence of a regular periodic function $w_{E}(\mathbf{y})$ such that:

$$
\mathbf{E}_{\perp}^{0}=\mathbf{E}_{\perp}+\nabla_{y} w_{E} .
$$


The function $w_{E}$ is the electrostatic potential associated with the microscopic electrostatic problem. Inserting (9) in equation (12) and projecting on both horizontal axis, we obtain:

$$
\operatorname{div}_{y}\left[\underline{\underline{\varepsilon}}_{\perp}^{0}\left(\mathbf{e}_{i}+\nabla_{y} w_{E, i}\right)\right]=0, i \in\{1,2\}
$$

where $\underline{\varepsilon}^{0}$ denotes the $2 \times 2$ matrix extracted from $\underline{\varepsilon}^{0}$ by removing the last line and last column. $\overline{\mathrm{B}} \mathrm{y}$ linearity, denoting $\mathbf{E}_{\perp}=\left(E_{1}, E_{2}\right)$, we derive that the potential $w_{E}$ is given by $w_{E}=E_{1} w_{E, 1}+E_{2} w_{E, 2}$, where $w_{E, i}$ are the periodic solutions of (13). We stress that these potentials depend upon y but also on $x_{3}$. In fact, we get a family of homogenization problems parametrized by the vertical coordinate. By (12) we obtain:

$$
\mathbf{E}^{0}(\mathbf{x}, \mathbf{y})=\mathcal{E}\left(\mathbf{y}, x_{3}\right) \mathbf{E}(\mathbf{x})
$$

where:

$$
\mathcal{E}\left(\mathbf{y}, x_{3}\right)=\left(\begin{array}{ccc}
1+\partial_{y_{1}} w_{E, 1} & \partial_{y_{1}} w_{E, 2} & 0 \\
\partial_{y_{2}} w_{E, 1} & 1+\partial_{y_{2}} w_{E, 2} & 0 \\
0 & 0 & 1
\end{array}\right)
$$

The magnetic field $\mathbf{H}^{0}$ can be handled in the same way since it satisfies exactly the same kind of equations as $\mathbf{H}^{0}$ (see (11)). In particular, we may represent its tranversal component in the form: $\mathbf{H}_{\perp}^{0}=\mathbf{H}_{\perp}+\nabla_{\perp} w_{H}$, where $w_{H}$ is a periodic magnetic potential (the possibility of this representation is due to the curl-free condition which means that no microscopic current is present). Analogously as in $(14,15)$, we find:

$$
\mathbf{H}^{0}(\mathbf{x}, \mathbf{y})=\mathcal{M}\left(\mathbf{y}, x_{3}\right) \mathbf{H}(\mathbf{x})
$$

where

$$
\mathcal{M}(\mathbf{y})=\left(\begin{array}{ccc}
1+\partial_{y_{1}} w_{H, 1} & \partial_{y_{1}} w_{H, 2} & 0 \\
\partial_{y_{2}} w_{H, 1} & 1+\partial_{y_{2}} w_{H, 2} & 0 \\
0 & 0 & 1
\end{array}\right)
$$

where:

$$
\operatorname{div}_{y}\left[\underline{\underline{\mu}}_{\perp}^{0}\left(\mathbf{e}_{i}+\nabla_{y} w_{H, i}\right)\right]=0, i \in\{1,2\}
$$

and $\underline{\mu}^{0}$ denotes the $2 \times 2$ matrix extracted from $\underline{\mu}^{0}$ by removing the last line and last column. Of course, the same remark as in the case of electric potentials holds: the functions $w_{H, i}$ depend on the vertical coordinate $x_{3}$. The above results show that, at the microscopic scale, the limit fields $\left(\mathbf{E}^{0}, \mathbf{H}^{0}\right)$ are completely determined by the physical fields $(\mathbf{E}, \mathbf{H})$. Now that the microscopic behavior is precised, we are able to determine the macroscopic system satisfied by $(\mathbf{E}, \mathbf{H})$. To that aim, let us choose a regular test function $\phi(\mathbf{x})$ independent of variable $\mathbf{y}$. From Maxwell equations we get

$$
\left\{\begin{array}{l}
\int_{\Omega} \mathbf{H}^{\eta}(\mathbf{x}) \cdot \operatorname{curl}(\phi) d \mathbf{x}=-i \omega \varepsilon_{0} \int_{\Omega} \underline{\underline{\varepsilon}}^{\eta}(\mathbf{x}) \mathbf{E}^{\eta}(\mathbf{x}) \phi(\mathbf{x}) d \mathbf{x} \\
\int_{\Omega} \mathbf{E}^{\eta}(\mathbf{x}) \cdot \operatorname{curl}(\phi) d \mathbf{x}=i \omega \mu_{0} \int_{\Omega} \underline{\underline{\mu}}^{\eta}(\mathbf{x}) \mathbf{H}^{\eta}(\mathbf{x}) \phi(\mathbf{x}) d \mathbf{x}
\end{array}\right.
$$

passing to the limit $\eta \rightarrow 0$, we get:

$$
\left\{\begin{array}{c}
\iint_{\Omega \times Y} \mathbf{H}^{0}(\mathbf{x}, \mathbf{y}) \cdot \operatorname{curl}(\phi) d \mathbf{x} d \mathbf{y}=-i \omega \varepsilon_{0} \iint_{\Omega \times Y} \underline{\underline{\varepsilon}}^{0}\left(\mathbf{y}, x_{3}\right) \mathbf{E}^{0}(\mathbf{x}, \mathbf{y}) \phi(\mathbf{x}) d \mathbf{x} d \mathbf{y} \\
\iint_{\Omega \times Y} \mathbf{E}^{0}(\mathbf{x}, \mathbf{y}) \cdot \operatorname{curl}(\phi) d \mathbf{x} d \mathbf{y}=i \omega \mu_{0} \iint_{\Omega \times Y} \underline{\underline{\mu}}^{0}\left(\mathbf{y}, x_{3}\right) \mathbf{H}^{0}(\mathbf{x}, \mathbf{y}) \phi(\mathbf{x}) d \mathbf{x} d \mathbf{y}
\end{array}\right.
$$

Recalling that $\left\langle\mathbf{E}^{0}\right\rangle=\mathbf{E}$ and that $\left\langle\mathbf{H}^{0}\right\rangle=\mathbf{H}$, we get:

$$
\left\{\begin{array}{l}
\operatorname{curl} \mathbf{E}=i \omega \mu_{0}\left\langle\underline{\underline{\mu}}^{0} \mathbf{H}^{0}\right\rangle \\
\operatorname{curl} \mathbf{H}=-i \omega \varepsilon_{0}\left\langle\underline{\underline{\underline{\varepsilon}}} \mathbf{E}^{0}\right\rangle
\end{array}\right.
$$


which, taking into account $(14,16)$, brings to the limit system:

$$
\left\{\begin{array}{l}
\operatorname{curl} \mathbf{E}=i \omega \mu_{0}\left\langle\underline{\underline{\mu}}^{0} \mathcal{M}\right\rangle\left(x_{3}\right) \mathbf{H} \\
\operatorname{curl} \mathbf{H}=-i \omega \varepsilon_{0}\left\langle\underline{\underline{\varepsilon}}^{0} \mathcal{E}\right\rangle\left(x_{3}\right) \mathbf{E}
\end{array}\right.
$$

\subsubsection{The special case of a $1 D$ grating}

Let us specialize the above results to the case of a one dimensional grating (fig. 3) that is, the pillars are invariant in the $x_{2}$ and $x_{3}$ directions (the basic cell $Y$ is depicted in fig. 3). We assume also that the pillars are made on a non-magnetic material and that the relative permittivity tensor is given by:

$$
\underline{\underline{\underline{\varepsilon}}}\left(y_{1}\right)=\left(\begin{array}{ccc}
\varepsilon_{1}\left(y_{1}\right) & 0 & 0 \\
0 & \varepsilon_{2}\left(y_{1}\right) & 0 \\
0 & 0 & \varepsilon_{3}\left(y_{1}\right)
\end{array}\right)
$$

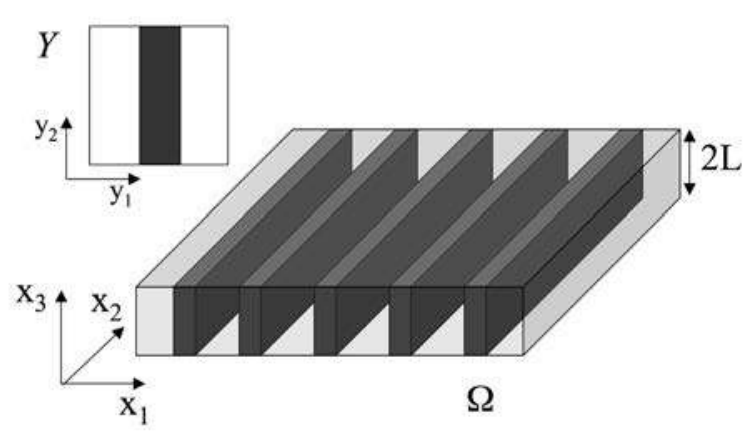

Fig. 3. Schematic of the 1D photonic crystal. The inset shows the basic cell $Y$.

The invariance of $\underline{\varepsilon}^{0}$ with respect to $y_{2}$ and the periodicity condition suggest that we look for solutions that are $y_{2}$-independent. Let us first consider $w_{E, 2}$ which satisfies: $\partial_{y_{1}}\left(\varepsilon_{1} \partial_{y_{1}} w_{E, 2}\right)=0$. This implies that $w_{E, 2}=$ cste. Next, we turn to $w_{E, 1}$. It satisfies: $\partial_{y_{1}}\left[\varepsilon_{1}\left(1+\partial_{y_{1}} w_{E, 1}\right)\right]=0$ Therefore $\varepsilon_{1}\left(1+\partial_{y_{1}} w_{E, 1}\right)=\mathrm{cste}=C$. Let us now average this relation, we get: $C\left\langle\frac{1}{\varepsilon_{1}}\right\rangle=$ $\left\langle 1+\partial_{y_{1}} w_{E, 1}\right\rangle$. Due to the periodicity of $w_{E, 1}$, we have: $\left\langle\partial_{y_{1}} w_{E, 1}\right\rangle=0$ and finally: $C=$ $\left\langle\frac{1}{\varepsilon_{1}}\right\rangle^{-1}$. The homogenized relative permittivity tensor is given by:

$$
\left\langle\underline{\underline{\varepsilon}}^{0} \mathcal{E}\right\rangle=\left(\begin{array}{ccc}
\left\langle\frac{1}{\varepsilon_{1}}\right\rangle^{-1} & 0 & 0 \\
0 & \left\langle\varepsilon_{2}\right\rangle & 0 \\
0 & 0 & \left\langle\varepsilon_{3}\right\rangle
\end{array}\right)
$$

We retrieve a well-established result concerning the homogenization of 1D photonic crystals [2].

\section{DISCUSSION}

The homogenized permeability and permittivity tensors are respectively $\left\langle\underline{\underline{\mu}} \underline{\underline{\mu}}^{0} \mathcal{M}\right\rangle$ and $\left\langle\underline{\underline{\varepsilon}}^{0} \mathcal{E}\right\rangle$. Both are matrix functions of the coordinate $x_{3}$. In case of cylindrical fibers (i.e. invariant along their axis), we would find that the effective tensors coincide with that obtained in the polarized cases $[2,3,15]$. In fact, because the permittivity and permeability tensors are not renormalized 
with respect to $\eta$, the homogenization process is purely local, that is, the effective constitutive relations are local ones. However, we emphasize that the locality is lost if we change the scale of the permittivity or permeability coefficients in the obstacles. In particular, the results obtained in the case of infinite conductivities in the polarized case [3] cannot be transposed to the case of fibers with finite length, due to the emergence of surprising non local effects which are studied in [16-18]. In this context, another problem is interesting: it is the study of the situation where the height $L$ of the pillar is small with respect to the free-space wavelength. An ansymptotic study can be performed in that case, leading to a simplified formulation of the diffraction problem [19].

We also note that an approach relying on explicit calculations, for instance using Blochwaves theory or Fourier-Bessel expansions, cannot work here, due the lack of an explicit representation of the fields in case of finite size fibers. The method that is used here proves to be particularly efficient, the results are obtained at the cost of very few calculations. Besides, it gives an interesting physical pictures of the homogenization process in terms of hidden variables: the homogenized parameters are obtained through clearly defined electrostatic and magnetostatic problems with respect to these variables. It should also be noted that the case of dielectric materials with losses is handled by our result. This result can be straightforwardly applied to the study of membrane photonic crystal in the low frequency range where phenomena of birefringence and dichroism are obtained [20,21].

In our homogenization result, it is clear that the main numerical problem is the solving of the annex problems $(13,18)$ for they give the effective matrices $\mathcal{E}$ and $\mathcal{M}$. In certain simple cases, for instance that of circular isotropic non magnetic rods and a permittivity constant in each connected region, it is possible to find an explicit expression for the effective permittivity (it is in fact a very old problem). However, for more complicated geometries, there is a general numerical procedure based on fictitious sources, that allows to solve both annex problems at a low numerical cost. It should also be stressed that if the wavelength is not very large with respect to the period, the device may still be homogenizable, but then the evanescent fields play an important role [22] that can make the effective parameters be dependent upon the environnement of the device.

\section{Acknowledgments}

This work was realized in the framework of the ANR project POEM PNANO 06-0030.

\section{References}

[1] J.B. Pendry, "Beyond metamaterials," Nature Mater. 5(10), 763-764 (2006) [doi:10.1038/nmat1740].

[2] A. Bensoussan, J.L. Lions, and G. Papanicolaou, Asymptotic Analysis for Periodic Structures, North-Holland, Amsterdam (1978).

[3] D. Felbacq and G. Bouchitté, "Homogenization of a set of parallel fibres," Waves in Random Media 7(2), 245-256 (1997) [doi:10.1088/0959-7174/7/2/006].

[4] D. Felbacq and G. Bouchitté, "Theory of mesoscopic magnetism in photonic crystals," Phys. Rev. Lett. 94, 183902 (2005) [doi:10.1103/PhysRevLett.94.183902].

[5] L. Peng, L. Ran, H. Chen, H. Zhang, J. A. Kong, and T. M. Grzegorczyk, "Experimental observation of left-handed behavior in an array of standard dielectric resonators," Phys. Rev. Lett. 98, 157403 (2007) [doi:10.1103/PhysRevLett.98.157403].

[6] D. Felbacq, G. Tayeb, and D. Maystre, "Localization of light by a set of parallel cylinder," J. Mod. Opt. 42(2) 473-482 (1995) [doi:10.1080/09500349514550421].

[7] D. Felbacq and G. Bouchitté, "Negative refraction in periodic and random photonic crystals," New J. Phys. 7, 159 (2005) [doi:10.1088/1367-2630/7/1/159]. 
[8] V. V. Poborchii, T. Tada, and T. Kanayama, "Si pillar photonic crystal slab with linear defects: transmittance and waveguide properties," Opt. Commun. 210(3-6), 285-290 (2002) [doi:10.1016/S0030-4018(02)01801-1].

[9] H. Benisty, C. Weisbuch, D. Labilloy, and M. Rattier, "Photonic crystals in twodimensions based on semiconductors: fabrication, physics and technology," Appl. Surf. Sci. 164(1-4), 205-218 (2000) [doi:10.1016/S0169-4332(00)00339-1]

[10] J. L. Erikson, D. Kinderlehrer, R. Kohn, and J.-L. Lions, Homogenization and Effective Moduli of Materials and Media, Springer-Verlag, New York (1986).

[11] R. C. McPhedran, L. C. Botten, and N. A. Nicorovici, "Homogenization of composites: dynamic and static theories," Physica B 279(1-3), 5-8 (2000) [doi:10.1016/S09214526(99)00653-5].

[12] R. C. McPhedran and N. A. Nicorovici, "Effective dielectric constant of arrays of elliptical cylinders," Physica A 241(1-2), 173-178 (1997) [doi:10.1016/S0378-4371(97)00078-2].

[13] D. Felbacq, "Anomalous homogeneous behaviour of metallic photonic crystals," J. Phys. A: Math. Gen. 33(4), 825-831(2000) [doi:10.1088/0305-4470/33/4/314].

[14] G. Allaire, "Homogenization and 2-scale convergence," SIAM J. Math. Anal. 23(6), 14821518 (1992) [doi:10.1137/0523084].

[15] S. Guenneau and F. Zolla, "Homogenization of 3D finite chiral photonic crystals," Phys. B Condens. Matter. 394(2), 145-147 (2007) [doi:10.1016/j.physb.2006.12.021].

[16] G. Bouchitté and D. Felbacq, "Homogenization of a wire photonic crystal: the case of small volume fraction," SIAM J. Appl. Math. 66(6), 2061-2084 (2006) [doi:10.1137/050633147].

[17] D. Felbacq and G. Bouchitté, "Left-handed media and homogenization of photonic crystals," Opt. Lett. 30(10), 1189-1191 (2005) [doi:10.1364/OL.30.001189].

[18] F. Zolla, D. Felbacq, and G. Bouchitté, "Bloch vector dependance of the plasma frequency in metallic photonic crystals," Phys. Rev. E 74, 056612 (2006) [doi:10.1103/PhysRevE.74.056612].

[19] H. Ammari, H. Kang, and F. Santosa, "Scattering of electromagnetic waves by thin dielectric planar structures," SIAM J. Math. Anal. 38(4), 1329-1342 (2007) [doi:10.1137/040618382].

[20] P. Halevi, A. A. Krokhin, and J. Arriaga, "Photonic crystal optics and homogenization of 2D periodic composites," Phys. Rev. Lett. 82, 719722 (1999) [doi:10.1103/PhysRevLett.82.719].

[21] P. Halevi, A. A. Krokhin, and J. Arriaga, "Long-wavelength limit (homogenization) for two-dimensional photonic crystals," Phys. Rev. B 65, 115208 (2002) [doi:10.1103/PhysRevB.65.115208].

[22] D. Felbacq and R. Smaâli, "Bloch modes dressed by evanescent waves and the generalized Goos-Hänchen effect in photonic crystals," Phys. Rev. Lett. 92, 193902 (2004) [doi:10.1103/PhysRevLett.92.193902]. 